\title{
CCAAT/enhancer-binding protein $\beta$ promotes receptor activator of nuclear factor-kappa-B ligand (RANKL) expression and osteoclast formation in the synovium in rheumatoid arthritis
}

\author{
Hidetoshi Tsushima, Ken Okazaki*, Kohei Ishihara, Takahiro Ushijima and Yukihide Iwamoto
}

\begin{abstract}
Introduction: CCAAT/enhancer-binding protein $\beta(C / E B P \beta)$ is a transcription factor that is activated in the synovium in rheumatoid arthritis (RA) and promotes expression of various matrix metalloproteinases. In this study, we examined whether C/EBP $\beta$ mediates the expression of receptor activator of nuclear factor-kappa-B ligand (RANKL) and drives osteoclast formation in primary fibroblast-like synoviocytes (FLS) from RA patients. The cooperation of C/EBP $\beta$ and activation transcription factor-4 (ATF4) in the regulation of the RANKL promoter was also investigated.

Methods: Immunofluorescence staining was performed for C/EBP $\beta$, RANKL, and ATF4 in synovium from RA patients. Adenovirus expression vectors for two major isoforms, C/EBP $\beta$-liver-enriched activator protein (LAP) and - liver-enriched inhibitory protein (LIP), or small interfering RNA for C/EBP $\beta$, were used to manipulate C/EBP $\beta$ expression in RA-FLS. RA-FLS over-expressing C/EBP $\beta$ were co-cultured with peripheral blood mononuclear cells (PBMCs) to test osteoclast formation by tartrate-resistant acid phosphatase (TRAP) staining. A promoter assay for RANKL, a chromatin immunoprecipitation (ChIP) assay and an immunoprecipitation (IP) assay were also performed.

Results: Immunofluorescence staining showed colocalization of C/EBP $\beta$, ATF4 and RANKL in RA synovium. Western blotting revealed the expression of C/EBP $\beta$-LAP and -LIP in RA-FLS. Over-expression of either C/EBPß-LAP or -LIP significantly increased the expression of RANKL mRNA, while C/EBPß-LIP down-regulated osteoprotegerin (OPG) mRNA. The RANKL/OPG mRNA ratio was significantly increased by C/EBP $\beta$-LIP over-expression. Knockdown of C/EBP $\beta$ with siRNA decreased the expression of RANKL mRNA. The number of TRAP-positive multinucleated cells was increased in co-cultures of PBMCs and FLS over-expressing either C/EBP $\beta$-LAP or -LIP, but was more significant with LIP. C/EBP $\beta$-LIP does not have a transactivation domain. However, promoter assays showed that C/EBP $\beta$-LIP and ATF4 synergistically transactivate the RANKL promoter. ChIP and IP assays revealed the cooperative binding of C/EBP $\beta$ and ATF4 on the RANKL promoter.
\end{abstract}

Conclusions: We demonstrated that C/EBP $\beta$, especially C/EBP $\beta$-LIP in cooperation with ATF4, is involved in osteoclast formation by regulating RANKL expression in RA-FLS. These findings suggest that C/EBP $\beta$ plays a crucial role in bone destruction in RA joints.

\footnotetext{
* Correspondence: okazaki@med.kyushu-u.ac.jp

Department of Orthopaedic Surgery, Graduate School of Medical Sciences,

Kyushu University, 3-1-1 Maidashi, Higashi-ku, Fukuoka 812-8582, Japan
} 


\section{Introduction}

Cartilage degeneration and bone destruction are the main features of rheumatoid arthritis (RA) [1]. Inflammation pathways are involved in the catabolic processes of articular cartilage and bone degeneration in RA. Inflammatory cytokines such as IL-1 $\beta$, TNF- $\alpha$, IL-6, and IL-17 play significant roles in mediating inflammation and joint destruction. These cytokines are expressed in arthritic joints in RA and induce expression of receptor activator of nuclear factor kappa B ligand (RANKL) in the synovium [2]. RANKL is an essential factor for osteoclast differentiation $[3,4]$. Osteoprotegerin (OPG) is a decoy receptor that inhibits RANKL activation of osteoclastogenesis and reduces bone resorption [5]. RA synovium-induced RANKL stimulates osteoclast differentiation at sites where bone and RA synovial membranes contact each other.

Inflammatory cytokines in RA joints activate numerous transcription factors including nuclear factor-kappa-B (NF-kB), activator protein-1 (AP-1), janus kinase-signal transducer and activator of transcription (JAK-STAT) and the CCAAT/enhancer-binding protein (C/EBP) family. The C/EBP family consists of six members: C/EBP $\alpha, \beta$, $\delta, \varepsilon, \gamma$, and $\zeta[6]$. C/EBP $\beta$ is an intron-less gene and has three major isoforms: $38 \mathrm{kD}$ (liver-enriched activator protein Star $(\mathrm{LAP} *)), 36 \mathrm{kD}$ (LAP) and $20 \mathrm{kD}$ (liverenriched inhibitory protein (LIP)) [7]. The isoforms, LAP* and LAP, each contain an $\mathrm{N}$-terminal transactivation domain (TAD) and a chromatin remodeling domain. The LIP isoform lacks the TAD, although it retains DNA binding capability, and is generally recognized to be a dominant negative isoform.

Recent studies indicated that $C / E B P \beta$ is involved in differentiation of osteoblasts and osteoclasts both physiologically and pathologically. C/EBP $\beta$ activates osteocalcin gene transcription and promotes osteoblast differentiation [8-10]. For osteoclast differentiation, the C/EBP $\beta$ isoform ratio in mononuclear cells regulates osteoclastogenesis through $\mathrm{V}$-maf musculoaponeurotic fibrosarcoma oncogene homolog B (MafB) [11]. C/EBP $\beta$ and RANKL are upregulated in GCT. C/EBP $\beta$ induces RANKL promoter activity in GCT stromal cells, which causes osteolysis [12]. In inflammatory chronic diseases such as RA, C/EBP $\beta$ is strongly induced in response to inflammatory stimulation. $C / E B P \beta$ is expressed in synovial tissues and chondrocytes of RA [13,14]. C/EBP $\beta$ plays a crucial role in cartilage degradation along with proteolytic enzymes such as matrix metalloproteinase-1 (MMP-1), MMP-3, MMP13, and aggrecanase- 2 (a disintegrin and metalloproteinase with thrombospondin motifs-5: ADAMTS-5) in inflammatory arthritis. Hence, we hypothesized that an imbalance of $\mathrm{C} / \mathrm{EBP} \beta$ isoforms may upset skeletal integrity in RA by being involved in both cartilage and bone destruction.

In this paper, we investigated whether C/EBP $\beta$ mediates the expression of RANKL in RA synovium and consequently, whether it induces osteoclast formation. In addition, we analyzed the mechanism of RANKL and OPG expression by the C/EBP $\beta$ isoforms, C/EBP $\beta$-LAP and -LIP, and by cooperation with activation transcription factor-4 (ATF4). Determining the mechanisms related to the regulation of RANKL expression and bone resorption by $\mathrm{C} / \mathrm{EBP} \beta$ may provide new insights into the development of potential therapies for RA patients.

\section{Methods \\ Clinical samples}

Tissue samples of synovium were obtained from patients with RA at the time of total knee arthroplasty (TKA) or synovectomy. Patients signed informed consent for providing tissue samples for this study. Subjects included seven RA patients (mean age, $60.3 \pm 11.3$ years), who fulfilled the 2010 American College of Rheumatology (ACR) and the European League Against Rheumatism (EULAR) diagnostic criteria for RA [15]. All studies were performed under the approval of the Institutional Ethics Board of Kyushu University (approval number: 22-99) and in accordance with the tenets of the Declaration of Helsinki.

\section{Isolation of human fibroblast-like synoviocytes}

Human fibroblast-like synoviocytes were isolated from the synovium of RA patients (RA-FLS). Synovial tissues were minced into small pieces and digested with $2 \mathrm{mg} / \mathrm{ml}$ collagenase L (Wako, Osaka, Japan) for 90 minutes at $37^{\circ} \mathrm{C}$. The collected cells were resuspended in DMEM supplemented with 10\% FBS (Gibco, Gaithersburg, MD, USA). Adherent cells were used after three to five passages.

As a control for RA-FLS, human fibroblast-like synoviocytes (HFLS, Cell Applications, San Diego, California, USA), which is a cell line derived from normal synovial tissue, were also cultured in DMEM supplemented with $10 \%$ FBS.

\section{Immunofluorescence staining}

Specimens were incubated overnight at $4^{\circ} \mathrm{C}$ with primary rabbit polyclonal anti-C/EBP $\beta$ antibodies (C-19; Santa Cruz Biotechnology, Santa Cruz, CA, USA) diluted 1:100, mouse monoclonal anti-RANKL antibodies (ab45039; Abcam, Cambridge, England) diluted 1:50, gout polyclonal anti-OPG antibodies (sc-8468; Santa Cruz Biotechnology) diluted 1:100, rabbit polyclonal anti-ATF4 antibodies (sc200; Santa Cruz Biotechnology) or normal rabbit IgG (sc-2027; Santa Cruz Biotechnology) diluted 1:100, respectively. RA-FLS plated on glass coverslips were transfected with adenovirus expression vectors for $\mathrm{C} /$ EBP $\beta$-LAP, -LIP or LacZ control [16] for 24 hours and then replaced with fresh medium. After 48 hours, immunofluorescence staining was performed. 


\section{Treatment of cells with cytokines}

Confluent cultures of RA-FLS were subjected to serumfree medium for 24 hours. This medium was replaced with fresh medium containing cytokines as follows: IL-1 $\beta$ (R\&D Systems, Minneapolis, MN, USA) at a concentration of $2 \mathrm{ng} / \mathrm{ml}$, TNF- $\alpha$ (Sigma-Aldrich, St Louis, MO, USA) at $10 \mathrm{ng} / \mathrm{ml}, \mathrm{IL}-6$ (R\&D Systems) at $10 \mathrm{ng} / \mathrm{ml}$, and IL-17 (R\&D Systems) at $100 \mathrm{ng} / \mathrm{ml}$. Cells were cultured for a further 48 hours. Concentrations of cytokines were determined based on previous literature [17-19].

\section{Western blotting}

Nuclear proteins were isolated using Nuclear and Cytoplasmic Extraction Reagent (NE-PER; Pierce, Rockford, IL, USA). Protein samples were transferred onto nitrocellulose membranes and were treated overnight at $4^{\circ} \mathrm{C}$ with primary antibodies.

RNA extraction and real-time reverse transcription (RT)-PCR Quantitative RT-PCR was performed with the LightCycler 2.0 system (Roche, Basel, Switzerland) using SYBR Premix Ex Taq (Takara Bio, Ohtsu, Japan). The primers were as follows: for C/EBP $\beta$, 5' -AGTACAAGATCCGGCGCGAG3' (sense) and 5' -TGCTTGAACAAGTTCCGCAG-3' (antisense); for RANKL, 5'-ATGAACTCCTTCTCCAC AAGCG-3' (sense) and 5'-CTCCTTTCTCAGGGCTG AG-3' (antisense; purchased from Takara Bio; oligo name HA137381F and R); for OPG, 5'-GCTTGAAACATA GGAGCTG-3' (sense) and 5'-GTTTACTTT GGT GCCAGG-3' (antisense); for ATF4, 5' -TCAAACCTCAT GGGTTCTCC-3' (sense) and 5'-GTGTCATCCAACGT GGTCAG-3' (antisense); and for GAPDH, 5'-GGTGAA GGTCGGAGTCAACGGA-3' (sense) and 5'-GAGGGAT CTCGCTCCTGGAAGA-3' (antisense). Data were normalized to the expression of GAPDH.

\section{Osteoclast formation in a peripheral blood mononuclear cell (PBMC) and RA-FLS co-culture system}

Peripheral blood was obtained from healthy donors. Isolated PBMCs $\left(2 \times 10^{5}\right.$ cells/well $)$ were resuspended in $\alpha$ minimum essential medium ( $\alpha$-MEM) containing 10\% FBS and $50 \mathrm{ng} / \mathrm{ml}$ macrophage colony-stimulating factor (MCSF; R\&D Systems) and then seeded in 96-well tissue culture plates. Three days later, adherent cells were used for the co-culture system.

Isolated FLS were transfected with adenovirus expression vectors for 24 hours and then fresh medium containing $10 \%$ FBS was added. After 48 hours, FLS were added into the 96-well plate with cultured PBMCs in $\alpha$-MEM containing $10 \%$ FBS and $50 \mathrm{ng} / \mathrm{ml}$ M-CSF. After 72 hours of co-culture, wells were stained for tartrate-resistant acid phosphatase (TRAP) (Primary Cell Co, Hokkaido, Japan). Osteoclasts were identified as TRAP-positive multinucleated cells that contained more than three nuclei.

\section{Gene knockdown in RA-FLS}

Predesigned small interference RNA (siRNA) for C/EBP $\beta$ (C/EBP $\beta$ siRNA-1 target sequence, 5'-CCCACGUGUAA CUGUCAGCtt-3' (sense) and 5' -GCUGACAGUUACAC GUGGGtt-3' (antisense)) or negative-control siRNA was purchased (Ambion, Austin, TX, USA). Transfection mixes were prepared using Lipofectamine 2000 (Invitrogen, Carlsbad, CA, USA). RA-FLS cells were cultured for 24 hours after transfection and then treated with $10 \mathrm{ng} / \mathrm{ml} \mathrm{IL-}$ $1 \beta$ for 72 hours.

\section{Human RANKL promoter reporter constructs}

Promoter constructs for human RANKL were sub-cloned into the pGL-4.10 (luc2) vector (Promega, Madison, WI, USA). The $5^{\prime}$-upstream region $(-1591 \mathrm{bp})$ of the human RANKL gene was prepared using human genomic DNA as a template (p-full). There are four putative binding sites for $\mathrm{C} / \mathrm{EBP} \beta$ between $-1591 \mathrm{bp}$ and $+12 \mathrm{bp}$. A 2-bp mutation (AA to $\mathrm{CC}$ ) was made at one site on the $\mathrm{p}$-full construct using the QuickChange site-directed mutagenesis kit (Stratagene, La Jolla, CA, USA).

\section{Plasmid transfection and luciferase assay}

HeLa cells seeded in 12-well plates were co-transfected with $0.5 \mu \mathrm{g} /$ well RANKL promoter constructs and various concentrations of pCMV-LAP, an expression vector of rat C/ EBP $\beta$-LAP directed by a cytomegalovirus promoter [20], or pCI-neo-LIP, an expression vector of rat C/EBP $\beta$-LIP [21], or pCMV6-AC-GFP-tagged ATF4 (OriGene, Rockville, MD, USA), an expression vector of human ATF4, using Lipofectamine LTX (Invitrogen). pRL-SV40 (Promega) was used as an internal control. Luciferase activity was then assayed using the Dual-luciferase Reporter Assay System (Promega).

\section{Chromatin immunoprecipitation (ChIP) assay}

RA-FLS cells were transfected with the adenovirus vector $\mathrm{C} /$ EBP $\beta$-LIP and incubated for 72 hours. A ChIP assay was performed with a ChIP Assay kit (Upstate Biotechnology, Lake Placid, NY, USA). The primers used in the PCR for RANKL promoter sequences were as follows: 5 '-GAGGGCGAAAG GAAGGAAGGGGAG-3' (sense) and 5'-GGCGTTGGA GAGCCCTGGCCTCGG -3' (antisense), which amplified between $-125 \mathrm{bp}$ and +26 bp. For a negative control, sequence between -1727 bp and -1487 bp was used. The PCR products were amplified for 33 cycles.

\section{Immunoprecipitation (IP)}

Nuclear proteins were isolated from RA-FLS transfected with adenovirus vector C/EBP $\beta$-LIP for 72 hours. The IP protocol used Dynabeads Protein A (Invitrogen). Anti-C/ EBP $\beta$ antibodies, anti-ATF4 antibodies or normal rabbit IgG and Dynabeads-complex, respectively, were added to antigen-containing lysates. Proteins were separated by SDS-PAGE and immunoblotted using specific antibodies. 


\section{Statistical analyses}

For in vitro investigations, nonparametric comparisons were performed using the Mann-Whitney $U$-test. $P$-values less than 0.05 were considered significant.

\section{Results \\ Co-localization of C/EBP $\beta$ and RANKL in the synovium from RA patients}

We initially examined C/EBP $\beta$ and RANKL expression by immunofluorescence staining in erosive areas of synovial tissue from RA patients. C/EBP $\beta$ and RANKL were expressed in RA synovial tissue (Figure 1A). The distribution patterns of C/EBP $\beta$ and RANKL were similar and both were strongly expressed in the synovial lining layer rather than in the sub-lining layer. The co-localization of $\mathrm{C} / \mathrm{EBP} \beta$ and RANKL in RA synovium suggests that $\mathrm{C} /$ $E B P \beta$ is involved in the regulation of RANKL expression.

\section{Expression of C/EBP $\beta$ in RA-FLS after treatment with pro-inflammatory cytokines}

Primary cultures of FLS were established and C/EBP $\beta$ expression was examined by western blotting. C/EBP $\beta$-LAP* (38 kDa), -LAP (36 kDa) and -LIP (20 kDa) were detected with LIP showing dominant expression. There was a varying degree of $\mathrm{C} / \mathrm{EBP} \beta$ expression (Figure 1B). The difference in expression levels of $\mathrm{C} / \mathrm{EBP} \beta$ may depend on the history of the patients such as degree of inflammation at the time of sample collection, disease duration, or therapies. Human FLS from normal articular joints lacks C/ EBP $\beta$ protein expression.

Next, we set out to determine whether pro-inflammatory cytokines could promote C/EBP $\beta$ protein in FLS. Western blots revealed that stimulation with IL-1 $\beta(2 \mathrm{ng} / \mathrm{ml})$, TNF- $\alpha$ (10 ng/ml), IL-6 (5 ng/ml), or IL-17 (100 ng/ml) increased the expression of both LAP and LIP isoforms in nuclear extracts, whereas the samples without any treatment did not show expression of $\mathrm{C} / \mathrm{EBP} \beta$ protein (Figure 1C). Interestingly, the expression of LIP was higher than that of LAP as shown in experiments of primary cultured RA-FLS.

\section{Overexpression of C/EBP $\beta$ regulates expression of RANKL and OPG in RA-FLS}

RA-FLS cells were transfected with adenovirus expression vectors expressing C/EBP $\beta$-LAP, -LIP or LacZ control. Western blots confirmed the exogenous overexpression of LAP or LIP in whole protein extracts isolated from transfected cells (Figure 2A). RANKL mRNA expression was examined by quantitative RT-PCR. The overexpression of LAP induced RANKL mRNA expression up to 80-fold compared to the LacZ control in a time-dependent manner. In RA-FLS transfected with the LIP vector, RANKL mRNA expression was increased approximately 6-fold (Figure 2A). We also investigated the expression of OPG.
Expression of OPG mRNA was upregulated by LAP in RA-FLS, whereas LIP significantly reduced OPG mRNA. Consequently, the RANKL-OPG ratio was highly upregulated in RA-FLS transfected with LIP (Figure 2B).

In addition, we examined whether $C / E B P \beta$ induced RANKL expression at the protein level by cell fluorescent immunostaining in a time-course experiment. The stimulated expression of $\mathrm{C} / \mathrm{EBP} \beta$ was observed in the nucleus of RA-FLS at 24 hours (Figure 2C). RANKL protein was localized in the cell cytoplasm of FLS over-expressing LAP or LIP at 72 hours. Similarly, the expression of OPG was also examined in a different series of experiments. The expression of OPG was stimulated by C/EBP $\beta$-LAP, but not by $\mathrm{C} / \mathrm{EBP} \beta$-LIP in RA-FLS at 72 hours.

\section{C/EBP $\beta$ knockdown by siRNA reduced RANKL expression in RA-FLS}

We assessed the effect of C/EBP $\beta$ knockdown on RANKL mRNA expression using siRNAs targeting C/EBP $\beta$ mRNA. Transfected cells were cultured with IL-1 $\beta$. C/EBP $\beta$ knockdown significantly reduced RANKL mRNA expression by $50 \%$ after IL-1 $\beta$ treatment in RA-FLS (Figure 2D).

\section{C/EBP $\beta$ induced osteoclast formation through RANKL expression in RA-FLS}

To investigate whether C/EBP $\beta$-induced RANKL expression stimulated osteoclast formation, we co-cultured RAFLS, which were transfected with adenovirus vectors expressing C/EBP $\beta$-LAP, -LIP or LacZ control, and PBMCs, which were isolated and stimulated with M-CSF. After three days of co-culture, formation of TRAP-positive multinucleated cells was observed in co-cultures of RAFLS over-expressing LAP or LIP, but not in control cells. Interestingly, more multinucleated cells were induced in the co-cultures of RA-FLS over-expressing LIP than with LAP transfected cells (Figure 3A). As a negative control, we performed monotype cell cultures of RA-FLS overexpressing $\mathrm{C} / \mathrm{EBP} \beta$, or $\mathrm{PBMC}$ over-expressing $\mathrm{C} / \mathrm{EBP} \beta$. These cells did not form osteoclasts (Figure 3B).

\section{C/EBP $\beta$ functions as an activator of the human RANKL promoter}

We further analyzed the in vitro promoter activity of human RANKL using HeLa cells. A luciferase reporter gene construct containing $-1591 \mathrm{bp}$ of the RANKL promoter was co-transfected with the expression vectors for $\mathrm{C} /$ EBP $\beta$-LAP (pCMV-LAP) or C/EBP $\beta$-LIP (pCI-neo-LIP) into HeLa cells. RANKL promoter activity was upregulated in a dose-dependent manner with either LAP or LIP (Figure 4A). To identify the $\mathrm{C} / \mathrm{EBP} \beta$ responsive element in the RANKL promoter, mutation analysis was performed using site-directed mutagenesis. We created four single mutation constructs in the RANKL promoter: single mutation 1 (mut-1), mutation 2 (mut-2), mutation 3 (mut-3), 


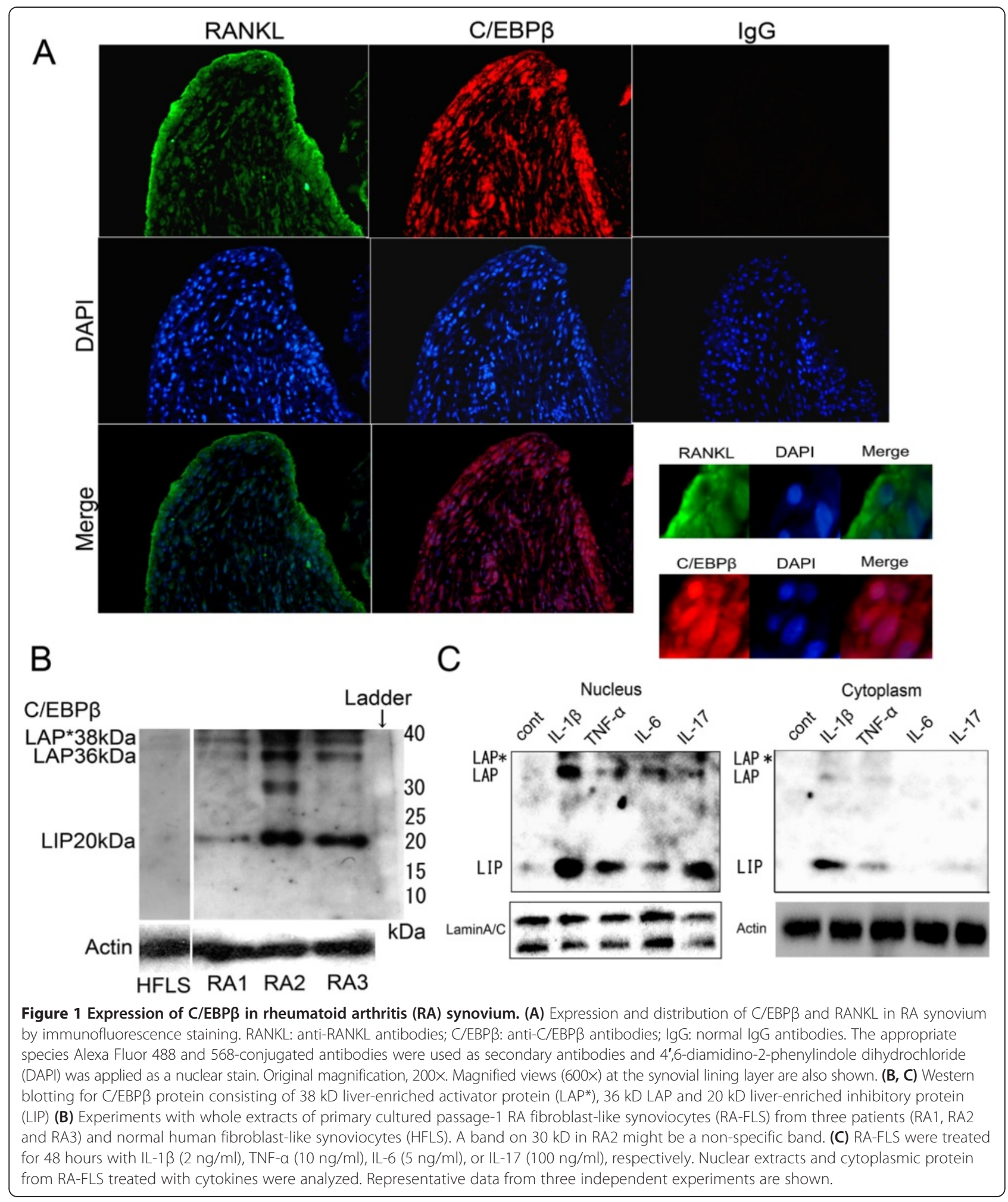

and mutation 4 (mut-4). Luciferase activities of mut-1, mut-2, and mut-3 reporter constructs were equally increased with pCMV-LAP, while mut- 4 reporter construct decreased luciferase activity by $30 \%$ (Figure $4 \mathrm{~B}$ ). Similarly, using the C/EBP $\beta$-LIP expression vector, mut- 2 and mut-3 did not show difference of activity from full reporter construct, while mut- 4 decreased to $25 \%$. These results showed that the putative C/EBP $\beta$ binding site is located between $-59 \mathrm{bp}$ and $-52 \mathrm{bp}$ in the RANKL promoter. The rationale for the increased activity of mut- 1 with $C / E B P \beta-$ 


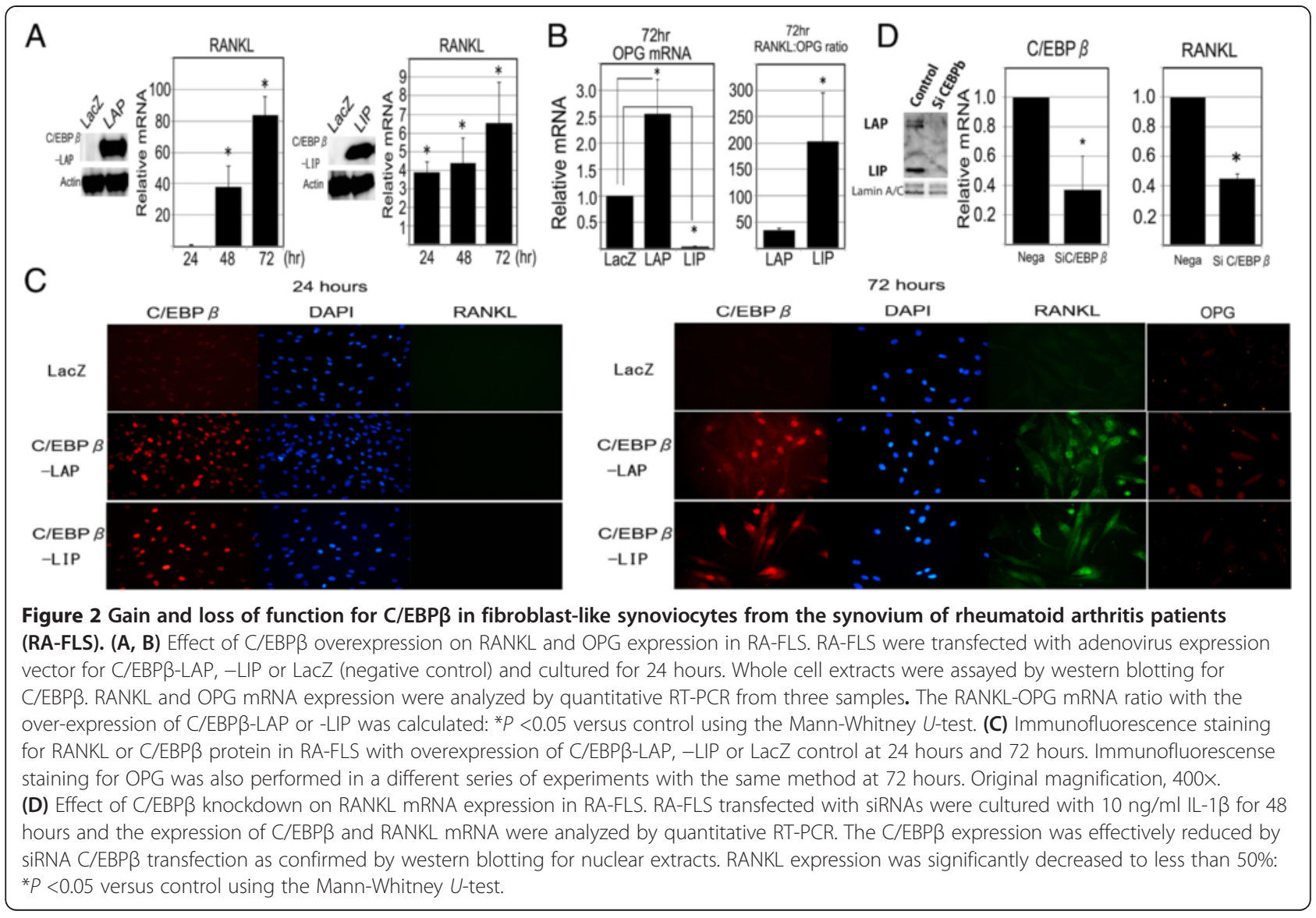

LIP expression vector has not been discovered yet as we failed to show the direct binding of $C / E B P \beta$ on this site.

\section{C/EBP $\beta$-LIP and ATF4 synergistically stimulate RANKL expression}

$\mathrm{C} / \mathrm{EBP} \beta$-LIP does not have an activation domain and is considered to be a dominant negative isoform. However, our results suggest that LIP is involved in RANKL expression in RA-FLS and plays a role in induction of osteoclast formation. We hypothesized that some transcriptional co-factors may cooperate with C/EBP $\beta$-LIP to activate transcription of the RANKL promoter. ATF4 is known to stimulate RANKL expression in osteoblasts [22]. Additionally, ATF4 has been shown previously to interact with $\mathrm{C} / \mathrm{EBP} \beta$, which activates various downstream factors such as osteocalcin and discoidin domain receptor tyrosine kinase (DDR2) [23]. Thus, we considered that a similar mechanism might exist for the regulation of RANKL gene expression. A luciferase assay showed that ATF4 slightly activated the RANKL promoter (Figure 4C). RANKL promoter activity was significantly enhanced following co-transfection of LIP and ATF4. Mut-4 abrogated the responsiveness of the RANKL promoter to the combination of LIP and ATF4. A ChIP assay was performed using RA-FLS over-expressing LIP and primers constructed from the human RANKL promoter sequence, which amplify sites including the $\mathrm{C} / \mathrm{EBP} \beta$ consensus site4. This analysis indicated that LIP binds to the RANKL promoter region containing CS-4 and that ATF4 also binds in the same region (Figure 4D). IP and immunoblotting demonstrated that ATF4 bound to over-expressed LIP in RA-FLS (Figure 4E). Collectively, these results suggest that $\mathrm{C} / \mathrm{EBP} \beta$-LIP cooperates with ATF4 in activating RANKL gene expression.

\section{ATF4 constitutively exists in RA synovium}

We then examined the localization of ATF4 in RA synovium. ATF4 was observed in erosive areas of RA synovium by immunofluorescence staining (Figure 5A). Western blotting showed that ATF4 was expressed in whole cell extracts of RA-FLS (Figure 5B). Next, we examined whether ATF4 expression was affected by $\mathrm{C} / \mathrm{EBP} \beta$ in RA-FLS transfected with adenovirus expression vectors in time-course experiments. ATF4 mRNA expression was not significantly changed by $\mathrm{C} / \mathrm{EBP} \beta$ (Figure $5 \mathrm{C}$ ). In addition, we performed organ cultures using RA synovium tissue. In RA synovium transfected with adenovirus LacZ control, ATF4 mainly localized in the cytoplasm of cells. Interestingly, in RA synovium over-expressing LIP, ATF4 was mainly located in the nucleus (Figure 5D). The results suggest that ATF4 


\section{A}

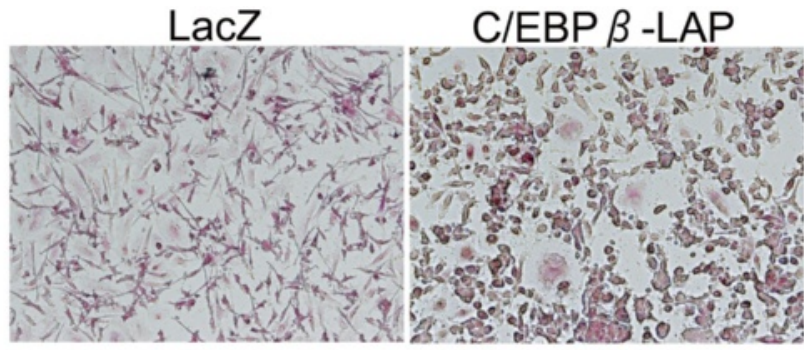

C/EBP $\beta-L I P$
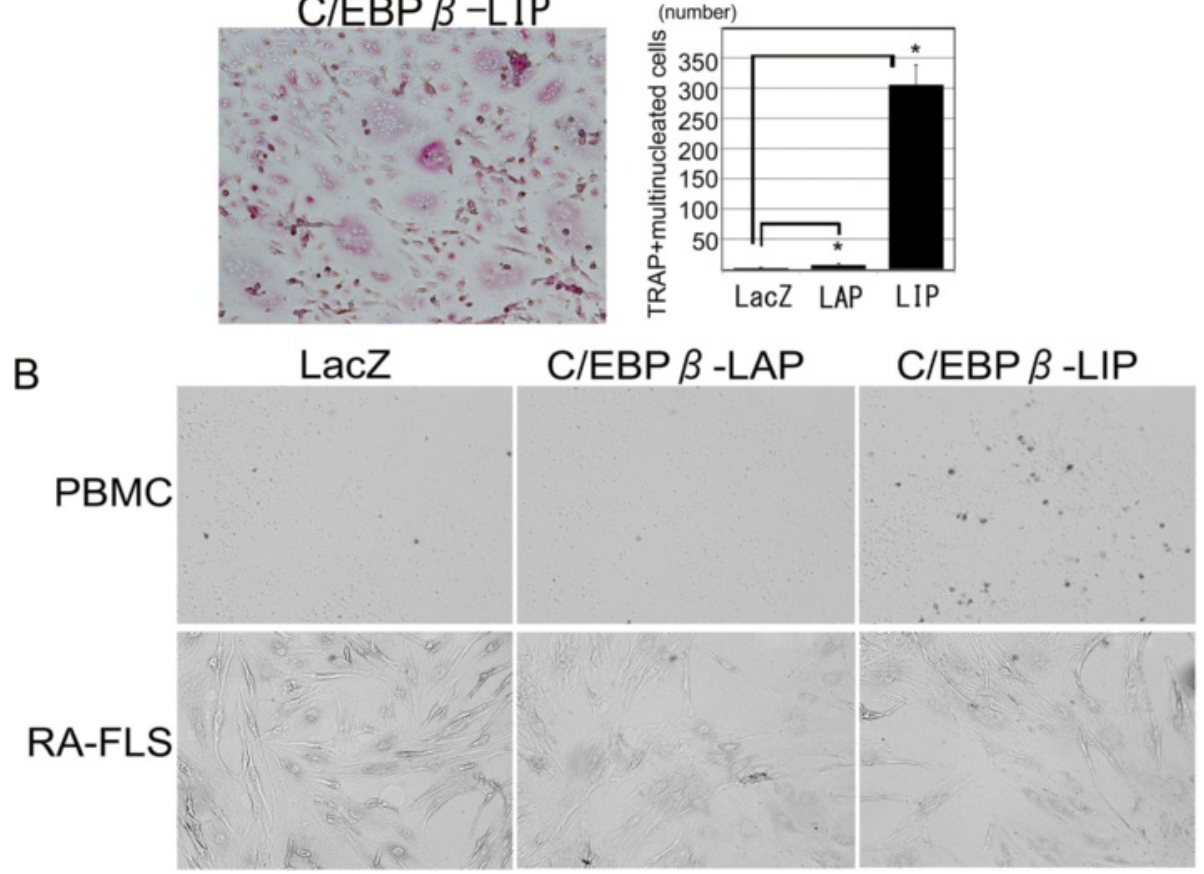

Figure 3 Osteoclast formation from peripheral blood mononuclear cells (PBMC) is promoted by the enhanced expression of C/EBP $\beta$ in fibroblast-like synoviocytes from the synovium of rheumatoid arthritis patients (RA-FLS). (A) Co-cultures of PBMCS and RA-FLS transfected with adenovirus vectors expressing C/EBP $\beta$-LAP, -LIP or LacZ control for 72 hours. Number of TRAP-positive multinucleated cells recognized in the co-culture system. Osteoclasts were identified as TRAP-positive multinucleated cells that contained more than three nuclei. Original magnification, 100x. ${ }^{*} P<0.05$ versus control using the Mann-Whitney U-test. (B) Negative control for the co-culture experiments. PBMC or RA-FLS over-expressing C/EBPß-LAP, -LIP or LacZ control, respectively, were cultured for 72 hours. No TRAP-positive multinucleated cells were observed.

is translocated from the cytoplasm into the nucleus in the RA synovium overexpressing C/EBP $\beta$-LIP although the mechanisms are not clear.

\section{Discussion}

In this study, we have shown that the transcription factor $\mathrm{C} / \mathrm{EBP} \beta$ promotes the expression of RANKL in RA synovium. C/EBP $\beta$-induced RANKL in synovium could induce the formation of osteoclasts. This paper demonstrates that RA-FLS expressed the C/EBP $\beta$-LIP isoform more dominantly than the C/EBP-LAP isoform in response to pro-inflammatory cytokines. C/EBP $\beta$-LIP stimulated RANKL expression even though C/EBP $\beta$-LIP lacks the transactivating domain. Recruitment of ATF4, which is constitutively expressed in the cytoplasm of RA-FLS, to the RANKL promoter might be the mechanism by which
C/EBP $\beta$-LIP activates the promoter. More interestingly, the effect of C/EBP $\beta$-LIP in osteoclast formation is stronger than that of C/EBP $\beta$-LAP. The lower expression of OPG might be involved in this mechanism. These results suggest that $C / E B P \beta$-LIP is one of the key regulators of inflammation-induced osteoclast formation. As $C / E B P \beta$ is also involved in cartilage degradation [19], C/EBP $\beta$ may play a crucial role in joint destruction in RA.

Understanding the mechanisms that mediate RANKL gene expression may aid development of new therapies for reducing bone resorption in RA. We showed that LAP and LIP directly bind a site located between $-59 \mathrm{bp}$ and $-52 \mathrm{bp}$ of the RANKL promoter. LIP is increased in the RA synovium and forms a complex with constitutively expressed ATF4. This complex may activate transcription of RANKL by binding to the C/EBP $\beta$ binding 


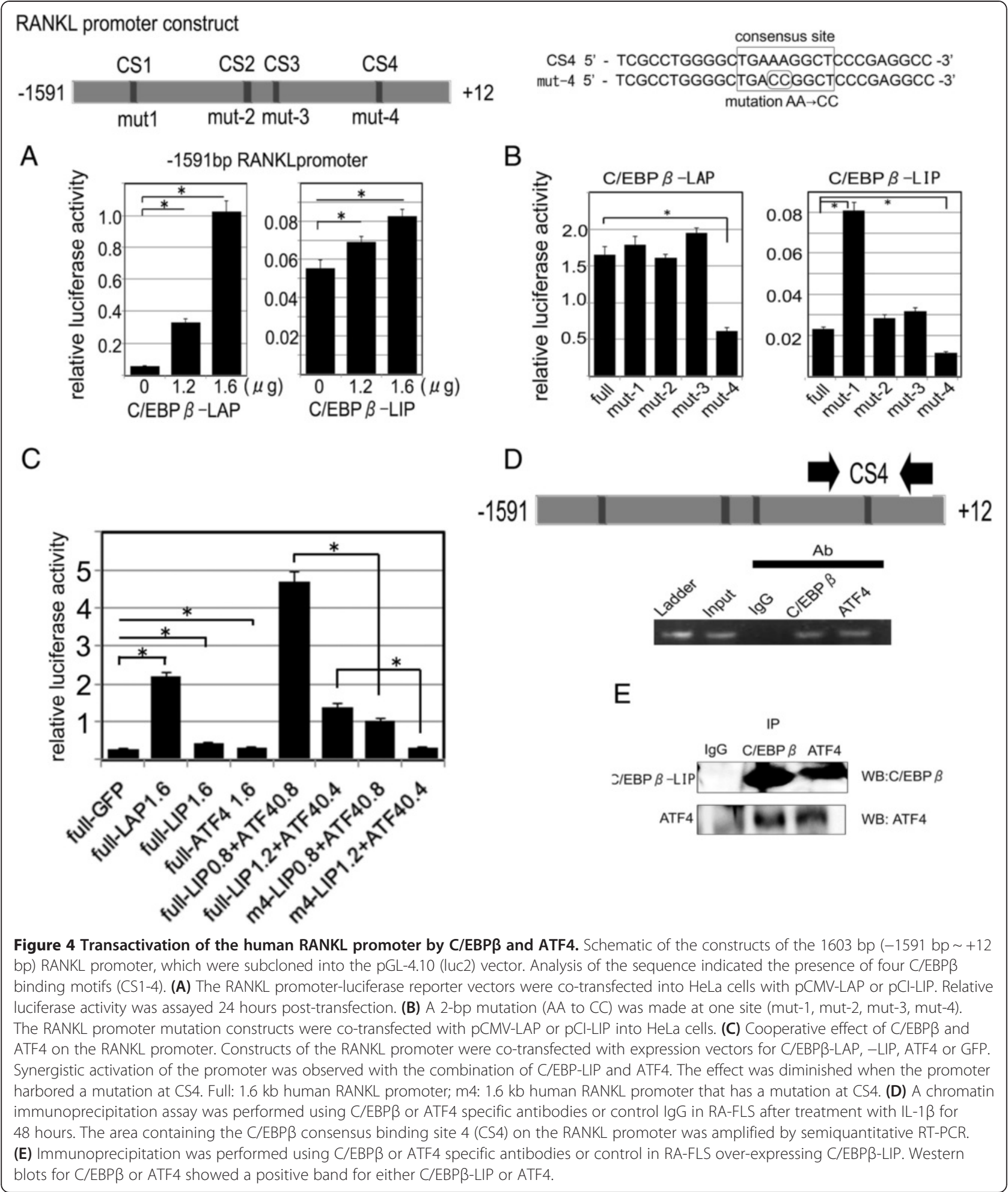

motif of the RANKL promoter. ATF4 belongs to the ATF/cAMP responsive element binding protein (CREB) family, which contains a basic leucine zipper region, and is one of the major regulators of osteoblast differentiation [24]. Moreover, ATF4 regulates RANKL expression [22].
This study showed that ATF4 is expressed in RA synovium and is involved in RANKL expression. Interestingly, in ex vivo experiments (Figure 5D), ATF4 was mainly expressed in the cytoplasm of FLS transfected with the LacZ adenovirus vector (control), while ATF4 tended to 


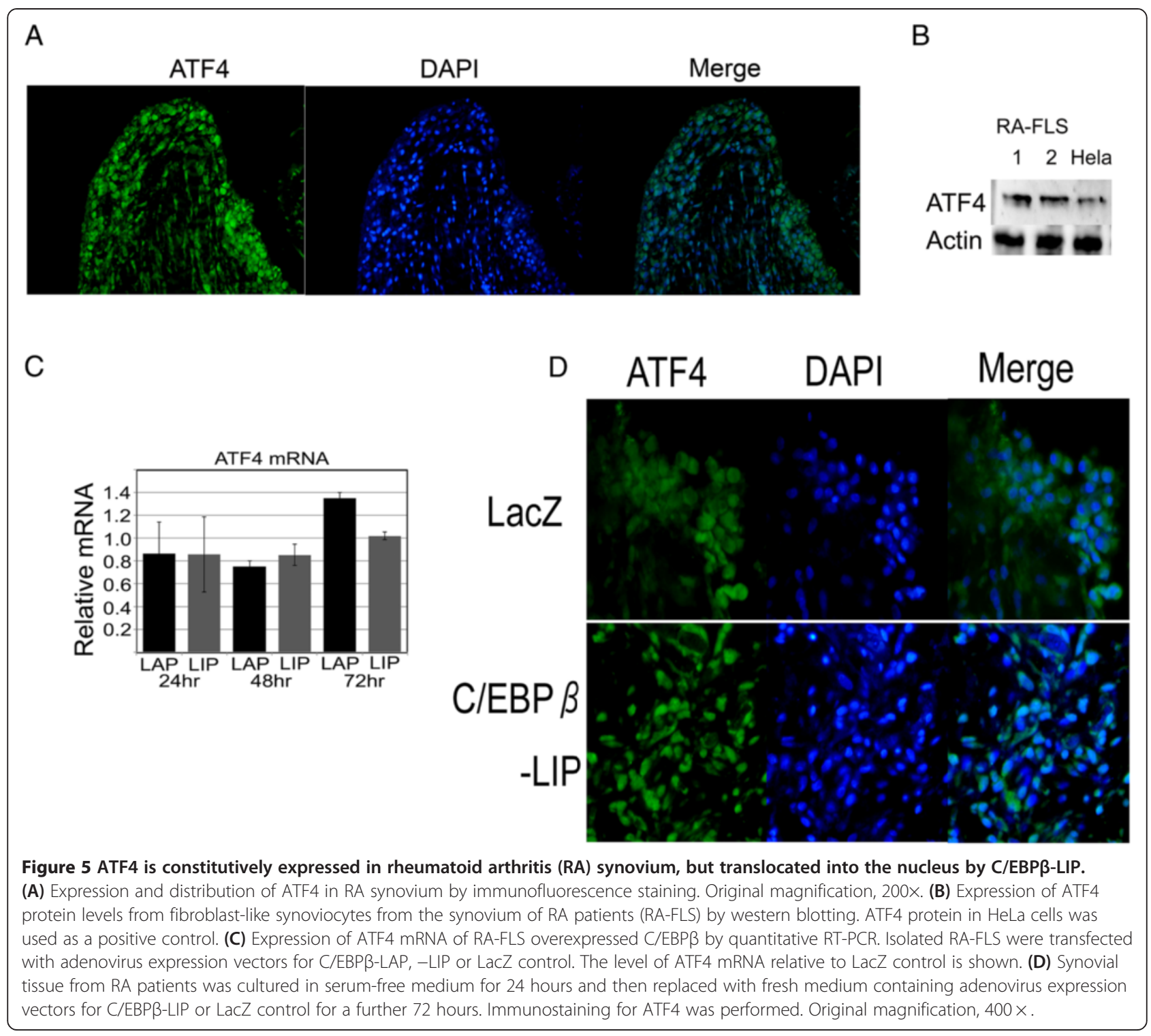

be located in the nucleus of FLS that overexpressed LIP. ATF4 in cooperation with $\mathrm{C} / \mathrm{EBP} \beta$ might be a crucial regulator of RANKL expression in mediating synoviuminduced bone resorption in RA. Other transcription factors, such as NF-kB, AP-1, STAT3 and Runt-related transcription factor-2 (Runx2), may also interface with $\mathrm{C} / \mathrm{EBP} \beta$. Runx2 and C/EBP $\beta$ cooperatively promote the expression of Indian Hedgehog in hypertrophic chondrocytes [25]. STAT3 is induced by IL-1 $\beta$, TNF- $\alpha$, and IL- 6 and increases the expression of IL- 6 and RANKL. A positive feedback loop, via IL-6 and STAT3, enhanced RANKL expression and osteoclastogenesis in inflammatory arthritis [26]. C/EBP $\beta$ was previously known as nuclear factor for IL-6 expression (NF-IL6) [27]. IL-6 induced by $\mathrm{C} / \mathrm{EBP} \beta$ regulates $\mathrm{C} / \mathrm{EBP} \beta$ gene transcription with recruitment of STAT3 to the promoter of the C/ EBP $\beta$ gene, especially in hepatocytes [28]. Therefore, we consider that positive feedback loops involving proinflammatory cytokines, IL-6, STAT3 and C/EBP $\beta$, might strongly increase RANKL expression in joints with RA. Indeed, STAT3 is essential for stimulation of RANKL and its binding element is located at $-82 \mathrm{bp}$ of the RANKL promoter, which is next to the $C / E B P \beta$ responsive motifs. C/EBP $\beta$-LIP may exist as an anchor to form a complex with other transcription factors mediated by inflammatory pathways.

The $C / E B P \beta$ isoform ratio can alter in response to cellular processes $[7,29,30]$. Of note, the LAP-LIP ratio is significant for osteoclastogenesis in PBMC through the mTOR pathway [11]. These papers indicate that an 
appropriate LAP-LIP ratio results in higher transcriptional activation of the target gene, which is very important in proliferation and differentiation. We showed that LIP protein is more highly expressed in RA-FLS than LAP. This imbalance of the LAP-LIP ratio caused a concomitant change in OPG mRNA expression. OPG is an endogenous inhibitor of RANKL-RANK interaction and is produced in synovial cells of patients with RA. The balance between levels of RANKL and OPG (RANKLOPG ratio) is correlated with the extent of bone resorption in RA joints [31]. The current study demonstrates that LIP drastically increased the RANKL-OPG ratio in RA-FLS, which subsequently induced significant osteoclast formation.

Previous studies showed that C/EBP $\beta$-LAP is a key regulator of cartilage degradation in inflammatory arthritis. C/EBP $\beta$-LAP plays a crucial role in cartilage degradation along with proteolytic enzymes such as MMP1, MMP-3, MMP-13, and aggrecanase-2 (ADAMTS-5) in chondrocytes and FLS in inflammatory arthritis $[19,32,33]$. The role of LIP is not well investigated in inflammatory arthritis. Our unpublished data revealed that overexpression of LIP in FLS increased MMP-1, MMP3, MMP-9, MMP-13, and ADAMTS-4 mRNA similar to the overexpression of LAP. The data presented here suggest that LAP and LIP coordinate in enhancing expression of RANKL, MMPs, and ADAMTSs, which may result in cartilage degradation and bone destruction of RA joints. C/EBP $\beta$ may be a common regulator, which can be stimulated in response to pro-inflammatory cytokines and upregulated in RA synovium. Therefore, selective blockage of C/EBP $\beta$ expression may be one potential strategy for preventing inflammation and bone resorption in arthritis.

This study has several limitations. First, double staining for C/EBP $\beta$ and RANKL did not work well on the RA synovium sections, although the reason was unclear. Therefore, we stained C/EBP $\beta$ and RANKL separately. However, the distribution of these molecules overlapped each other, suggesting that C/EBP $\beta$ and RANKL are coexpressed. Next, the promoter assays were performed in HeLa cells. We could not obtain reliable results of the promoter assay in RA FLS probably because of low transfection efficiency. However, the purpose of these experiments was to see the effect of various transcription factors that were exogenously introduced by expression vectors. Therefore, the influence of cell characteristics on the results is considered to be limited. Thirdly, the promoter that harbors a mutation in CS1 showed increased activity with C/EBP $\beta$-LIP (Figure 4B). This result suggests that LIP act as a repressor in CS1. However, ChIP assay for CS1 sequences did not show binding of C/EBP $\beta$ on CS1 (data not shown). Therefore, the function of CS1 remains unclear at the moment.

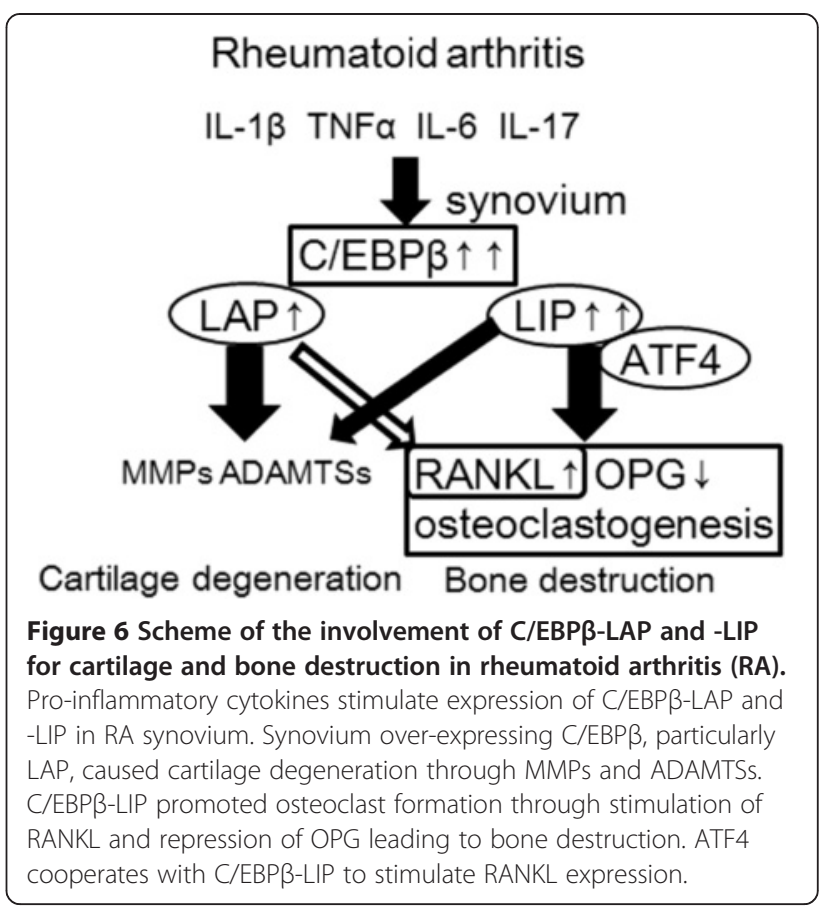

\section{Conclusions}

In conclusion, $\mathrm{C} / \mathrm{EBP} \beta$ increased RANKL expression in RA-FLS and induced osteoclastogenesis (Figure 6). Proinflammatory cytokines significantly induced C/EBP $\beta$-LIP, which strongly induced osteoclastogenesis by increasing the RANKL-OPG ratio in RA-FLS. LIP possesses transactivation activity for the RANKL promoter by recruiting ATF4, which constitutively exists in the cytoplasm of RAFLS, to the C/EBP binding site in the RANKL promoter. In pathological inflammatory arthritis, $C / E B P \beta$ is a crucial factor in damaging cartilage and bone in joints.

\section{Abbreviations}

a-MEM: a-minimum essential medium; AP-1: activator protein-1; ATF-4: activation transcription factor 4; bp: base pairs; C/EBP: CCAAT/enhancer binding protein; ChIP: chromatin immunoprecipitation; DMEM: Dulbecco's modified Eagle's medium; FBS: fetal bovine serum; FLS: fibroblast-like synoviocytes; GCT: giant cell tumor; IL: interleukin; JAK-STAT: janus kinase-signal transducer and activator of transcription; LAP: liver-enriched activator protein; LIP: liver-enriched inhibitory protein; M-CSF: macrophage colony-stimulating factor; MMP: matrix metalloproteinase; NF-kB: nuclear factor-kappa-B; OPG: osteoprotegerin; PBMC: peripheral blood mononuclear cell; RA: rheumatoid arthritis; RA-FLS: fibroblast-like synoviocytes from the synovium of RA patients; RANKL: receptor activator of nuclear factor kappa B ligand; RT: reverse transcription; siRNA: small interference RNA; TNF: tumor necrosis factor; TAD: N-terminal transactivation domain; TRAP: tartrate-resistant acid phosphatase.

\section{Competing interests}

The authors declare that they have no competing interests.

\section{Authors' contributions}

$\mathrm{HT}$ participated in all the experiments and drafted the manuscript. KO conceived of the study, and participated in its design and coordination and helped to draft the manuscript. Kl participated in the western blotting for RA-FLS and immunofluorescense staining of cells for OPG and helped to revise the manuscript. TU participated in the knockdown experiment with 
siRNA and the luciferase assays and helped to draft the manuscript. YI participated in the design of the study and involved in revising the manuscript critically for important intellectual content. All authors read and approved the final manuscript.

\section{Acknowledgements}

This work was supported in part by a Grant-in-Aid for Scientific Research (Basic Research (C), (number 23592218) from the Japan Society for the Promotion of Science and a Grant for Research on Regenerative Medicine for Clinical Application (number 26220101) from the Ministry of Health, Labour and Welfare of Japan. The funders had no role in the study design, data collection and analysis, decision to publish, or preparation of the manuscript.

\section{Received: 15 August 2014 Accepted: 19 January 2015}

\section{Published online: 17 February 2015}

\section{References}

1. Firestein GS. Evolving concepts of rheumatoid arthritis. Nature 2003:423:356-61.

2. Takayanagi H, Oda H, Yamamoto S, Kawaguchi H, Tanaka S, Nishikawa T, et al. A new mechanism of bone destruction in rheumatoid arthritis: synovial fibroblasts induce osteoclastogenesis. Biochem Biophys Res Commun. 1997;240:279-86.

3. Yasuda H, Shima N, Nakagawa N, Yamaguchi K, Kinosaki M, Mochizuki S, et al. Osteoclast differentiation factor is a ligand for osteoprotegerin/ osteoclastogenesis-inhibitory factor and is identical to TRANCE/RANKL. Proc Natl Acad Sci USA. 1998;95:3597-602

4. Lacey DL, Timms E, Tan HL, Kelley MJ, Dunstan CR, Burgess T, et al. Osteoprotegerin ligand is a cytokine that regulates osteoclast differentiation and activation. Cell. 1998;93:165-76.

5. Tsuda E, Goto M, Mochizuki S, Yano K, Kobayashi F, Morinaga T, et al. Isolation of a novel cytokine from human fibroblasts that specifically inhibits osteoclastogenesis. Biochem Biophys Res Commun. 1997;234:137-42.

6. Cao Z, Umek RM, McKnight SL. Regulated expression of three C/EBP isoforms during adipose conversion of 3T3-L1 cells. Genes Dev. 1991:5:1538-52.

7. Descombes P, Schibler U. A liver-enriched transcriptional activator protein, LAP, and a transcriptional inhibitory protein, LIP, are translated from the same mRNA. Cell. 1991;67:569-79.

8. Gutierrez S, Javed A, Tennant DK, van Rees M, Montecino M, Stein GS, et al. CCAAT/enhancer-binding proteins (C/EBP) beta and delta activate osteocalcin gene transcription and synergize with Runx2 at the C/EBP element to regulate bone-specific expression. J Biol Chem. 2002;277:1316-23.

9. Tominaga H, Maeda S, Hayashi M, Takeda S, Akira S, Komiya S, et al. CCAAT/ enhancer-binding protein beta promotes osteoblast differentiation by enhancing Runx2 activity with ATF4. Mol Biol Cell. 2008;19:5373-86.

10. Hata K, Nishimura R, Ueda M, Ikeda F, Matsubara T, Ichida F, et al. A CCAAT/ enhancer binding protein beta isoform, liver-enriched inhibitory protein, regulates commitment of osteoblasts and adipocytes. Mol Cell Biol. 2005:25:1971-9.

11. Smink JJ, Begay V, Schoenmaker T, Sterneck E, de Vries TJ, Leutz A. Transcription factor C/EBPbeta isoform ratio regulates osteoclastogenesis through MafB. EMBO J. 2009;28:1769-81.

12. Ng PK, Tsui SK, Lau CP, Wong CH, Wong WH, Huang L, et al. CCAAT/ enhancer binding protein beta is up-regulated in giant cell tumor of bone and regulates RANKL expression. J Cell Biochem. 2010:110:438-46.

13. Pope RM, Lovis R, Mungre S, Perlman H, Koch AE, Haines 3rd GK. C/EBP beta in rheumatoid arthritis: correlation with inflammation, not disease specificity. Clin Immunol. 1999;91:271-82.

14. Nishioka K, Ohshima S, Umeshita-Sasai M, Yamaguchi N, Mima T, Nomura S, et al. Enhanced expression and DNA binding activity of two CCAAT/enhancerbinding protein isoforms, C/EBPbeta and C/EBPdelta, in rheumatoid synovium. Arthritis Rheum. 2000:43:1591-6.

15. Aletaha D, Neogi T, Silman AJ, Funovits J, Felson DT, Bingham 3rd CO, et al. Rheumatoid arthritis classification criteria: an American College of Rheumatology/European League Against Rheumatism collaborative initiative. Arthritis Rheum. 2010:2010:2569-81.

16. Sakaue H, Konishi M, Ogawa W, Asaki T, Mori T, Yamasaki M, et al. Requirement of fibroblast growth factor 10 in development of white adipose tissue. Genes Dev. 2002;16:908-12.
17. Tunyogi-Csapo M, Kis-Toth K, Radacs M, Farkas B, Jacobs JJ, Finnegan A, et al. Cytokine-controlled RANKL and osteoprotegerin expression by human and mouse synovial fibroblasts: fibroblast-mediated pathologic bone resorption. Arthritis Rheum. 2008;58:2397-408.

18. Hashizume M, Hayakawa N, Mihara M. IL-6 trans-signalling directly induces RANKL on fibroblast-like synovial cells and is involved in RANKL induction by TNF-alpha and IL-17. Rheumatology (Oxford). 2008;47:1635-40.

19. Tsushima H, Okazaki K, Hayashida M, Ushijima T, Iwamoto Y. CCAAT/ enhancer binding protein beta regulates expression of matrix metalloproteinase-3 in arthritis. Ann Rheum Dis. 2012;71:99-107.

20. Descombes P, Chojkier M, Lichtsteiner S, Falvey E, Schibler U. LAP, a novel member of the C/EBP gene family, encodes a liver-enriched transcriptional activator protein. Genes Dev. 1990:4:1541-51.

21. Zahnow CA, Cardiff RD, Laucirica R, Medina D, Rosen JM. A role for CCAAT/ enhancer binding protein beta-liver-enriched inhibitory protein in mammary epithelial cell proliferation. Cancer Res. 2001;61:261-9.

22. Elefteriou F, Ahn JD, Takeda S, Starbuck M, Yang X, Liu X, et al. Leptin regulation of bone resorption by the sympathetic nervous system and CART. Nature. 2005;434:514-20.

23. Lin KL, Chou CH, Hsieh SC, Hwa SY, Lee MT, Wang FF. Transcriptional upregulation of DDR2 by ATF4 facilitates osteoblastic differentiation through p38 MAPK-mediated Runx2 activation. J Bone Miner Res. 2010;25:2489-503.

24. Yang X, Matsuda K, Bialek $P$, Jacquot $S$, Masuoka HC, Schinke T, et al. ATF4 is a substrate of RSK2 and an essential regulator of osteoblast biology; implication for Coffin-Lowry Syndrome. Cell. 2004;117:387-98.

25. Ushijima T, Okazaki K, Tsushima H, Ishihara K, Doi T, Iwamoto Y. CCAAT/ enhancer binding protein $\beta$ regulates expression of indian hedgehog during chondrocytes differentiation. Plos One. In press 2014.

26. Mori T, Miyamoto T, Yoshida H, Asakawa M, Kawasumi M, Kobayashi T, et al. IL-1 beta and TNFalpha-initiated IL-6-STAT3 pathway is critical in mediating inflammatory cytokines and RANKL expression in inflammatory arthritis. Int Immunol. 2011:23:701-12.

27. Akira S, Isshiki H, Sugita T, Tanabe O, Kinoshita S, Nishio $Y$, et al. A nuclear factor for IL-6 expression (NF-IL6) is a member of a C/EBP family. EMBO J. 1990;9:1897-906

28. Niehof M, Streetz K, Rakemann T, Bischoff SC, Manns MP, Horn F, et al. Interleukin-6-induced tethering of STAT3 to the LAP/C/EBPbeta promoter suggests a new mechanism of transcriptional regulation by STAT3. J Biol Chem. 2001;276:9016-27.

29. Calkhoven CF, Muller C, Leutz A. Translational control of C/EBPalpha and C/EBPbeta isoform expression. Genes Dev. 2000;14:1920-32.

30. Ushijima T, Okazaki K, Tsushima H, Iwamoto Y. CCAAT/enhancer-binding protein beta regulates the repression of type II collagen expression during the differentiation from proliferative to hypertrophic chondrocytes. J Biol Chem. 2014;289:2852-63.

31. Haynes DR, Crotti TN, Loric M, Bain Gl, Atkins GJ, Findlay DM. Osteoprotegerin and receptor activator of nuclear factor kappaB ligand (RANKL) regulate osteoclast formation by cells in the human rheumatoid arthritic joint. Rheumatology (Oxford). 2001;40:623-30.

32. Raymond L, Eck S, Mollmark J, Hays E, Tomek I, Kantor S, et al. Interleukin-1 beta induction of matrix metalloproteinase-1 transcription in chondrocytes requires ERK-dependent activation of CCAAT enhancer-binding protein-beta. J Cell Physiol. 2006;207:683-8.

33. Hayashida M, Okazaki K, Fukushi J, Sakamoto A, Iwamoto Y. CCAAT/ enhancer binding protein beta mediates expression of matrix metalloproteinase 13 in human articular chondrocytes in inflammatory arthritis. Arthritis Rheum. 2009;60:708-16. 